\title{
Análisis de tecnologías para la transformación de material aglomerado de madera con fines de reciclaje
}

\section{Analysis of technologies for the transformation of agglomerated wood material for recycling purposes}

\section{Aura Karina Reyes Echeverría 1.; Luis Eduardo Bautista Rojas 2.; Luis Carlos Quintero Cárdenas ${ }^{3}$}

\author{
${ }^{1}$ Maestría en Ciencias y Tecnologías Ambientales, Universidad Santo Tomás, seccional Bucaramanga. \\ akreyes42@gmail.com \\ ${ }^{2}$ Profesor, Universidad Industrial de Santander. Grupo de investigación Interfaz. \\ luis.bautista@correo.uis.edu.co \\ ${ }^{3}$ Diseñador Industrial, lucas.quintero@gmail.com
}

\begin{abstract}
Resumen
Los Tableros de Partículas y Tableros de Fibra de madera están compuestos de agua y aglomerantes como la urea-formaldehido que hacen su proceso de degradación más lento. En la transformación de tablero se generan residuos que son utilizados como combustible o depositados en vertederos municipales, lo cual es perjudicial para el medio ambiente debido a las sustancias químicas nocivas utilizadas en el proceso de adhesión y conformación del tablero. El objetivo de este trabajo es realizar una búsqueda de tecnologías para la transformación de los residuos de material aglomerado de madera, con fines de reúso o reciclaje. Se realizó una identificación de palabras clave, se elaboró una ecuación de búsqueda, se ejecutó en dos bases de datos científicas y en webgrafía. Los resultados fueron analizados para orientar la aplicación de los procesos de transformación. Como resultados se analizaron 31 artículos de investigación, 6 casos de uso, se concluyen 7 procesos químicos y 5 procesos físicos.
\end{abstract}

Palabras clave: madera, medio ambiente, proceso, residuos.

\begin{abstract}
Particle Boards and Wood Fiber Boards are composed of water and binders such as ureaformaldehyde that make their degradation process slower. In the board transformation, waste is generated that is used as fuel or deposited in municipal landfills, which is harmful to the environment due to the harmful chemical substances used in the process of adhesion and conformation of the board. The objective of this work is to carry out a search of technologies for the transformation of the waste of agglomerated wood material, for reuse or recycling purposes. Identification of key words was carried out, a search equation was developed, it was executed in two scientific databases and in webgraphy. The results were analyzed to guide the application of the transformation processes. As results, 31 research articles were analyzed, 6 cases of use, 7 chemical processes and 5 physical processes were concluded.
\end{abstract}

Keywords: Environment, Process, Waste, Wood. 
55

\section{Introducción}

En el sector del mueble y la madera, se generan residuos de materia prima provenientes de los procesos de transformación de tableros aglomerados. Estos tienen una conformación fisico-química de diferentes especies de madera, y sustancias químicas como adhesivos y aditivos para proveer las propiedades necesarias para su uso en la industria (Declaration, 2017). Entre estos el contenido de formaldehido; conocido por ser un agente antiséptico y tóxico, hace más difícil la biodegradabilidad de los tableros para los microorganismos (Costa \& Teixeira, 2015).

En una revisión de campo en el sector del mueble y la madera de Bucaramanga, se establece que en la empresa Madecentro Colombia S.A.S se presenta una problemática de residuos generados por la transformación de tableros aglomerados, entre estos se encuentran, (Fronti de García \& Cuesta Fernández, 2007):

- Impacto Ambiental en la generación de residuos de materia prima, sus efectos en la naturaleza, agotamiento de la capa de ozono, calentamiento global, o la eutroficación.

- Afectación económica en nula rentabilidad del residuo sólido por desconocimiento de tecnologías y prácticas como el reciclaje para generación de nuevos productos.

- Sanciones ambientales a las cuales está expuesta la empresa por normatividad y legislación ambiental.

- Área de almacenamiento donde se incrementa el espacio de bodegaje por acumulación de residuos.

- Afectación social y salud pública estimada por la generación de plagas, enfermedades y basuras para vecinos y trabajadores.

- Perdida de recurso energético invertido en el proceso de generación del tablero y costo medio ambiental por recursos naturales invertidos en el proceso.

En Colombia la empresa Primadera, tiene como opción de materia prima la madera urbana o madera proveniente del reciclaje. Es una empresa pionera en el país en este desarrollo, contribuyendo a la preservación del medio ambiente y generando un impacto social positivo, (Primadera, 2016)

También, en el Reino Unido, el BioComposites Center (Center, 2018) estudia el desarrollo de un proceso donde es posible reciclar MDF de desechos en MDF "nuevo" utilizando tecnología de microondas para reprocesar las fibras; la clave fue el procesamiento continuo que no dañó las fibras, (Panel, 2012).

Este proyecto se realiza por fases, en la fase 1, se realiza una recolección de información de las tecnologías actualmente utilizadas en el mundo para la transformación de material aglomerado, se revisan procesos, maquinaria y productos obtenidos. En la fase 2, se realiza un resumen de información relevante de los procesos e implementaciones de tecnologías realizadas en el mundo.

El propósito de este proyecto es analizar las tecnologías de transformación de tableros aglomerados en el mundo, y beneficiar a la empresa Madecentro Colombia S.A.S y a la población en general, que se ven afectados por los impactos ambientales de los residuos sólidos generan, como aumento del calentamiento global.

Entonces este documento se elabora de la siguiente manera, en el numeral 2 se expone la metodologia utilizada, y el desarrollo de la misma en tres puntos, la busqueda de la información, analisis de la información y la observacion de campo, por otro lado, en el 
56

numeral 3 se presentan los resultados y por último las conclusiones del trabajo realizado.

\section{Metodología}

Para la ejecución de esta revisión se plantea un diseño metodológico realizado en 2 fases:

Fase 1: Búsqueda, Definir ecuación de búsqueda mediante palabras claves y términos de búsqueda, filtrado de artículos de investigación relevantes en el tema, y búsqueda de casos de uso en el mundo.

Fase 2: Análisis de información, Analizar en la literatura aspectos del proceso de reciclaje de láminas de madera procesada, propiedades de las físico-química de los tableros, tecnologías y procesos utilizados alrededor del mundo para la transformación de material residuo de laminas de madera procesada.

\subsection{Revisión}

Se definieron dos bases de datos para la realización de la investigación, y una búsqueda en Google Académico, delimitando por 4 años antigüedad de los artículos, de lo cual se extraen 31 artículos en total y 6 casos de uso, Figura 1.

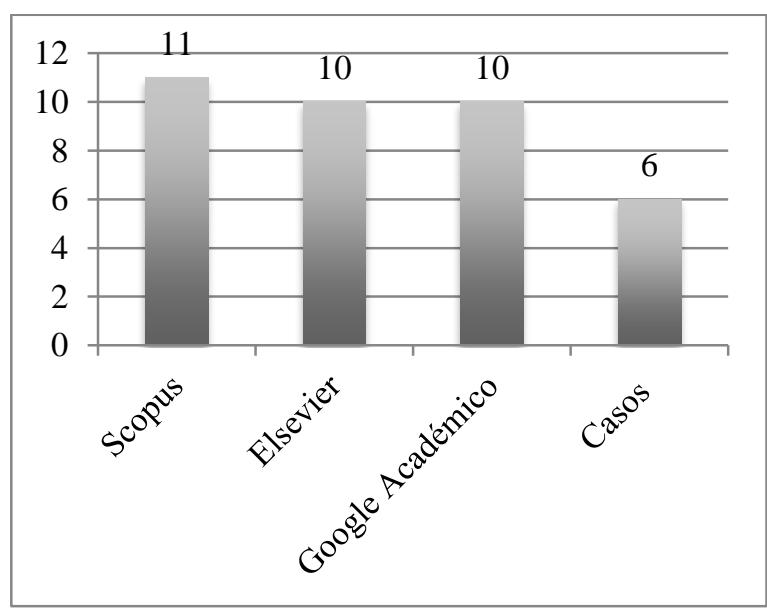

Fig. 1 Número de artículos seleccionados por sistema de búsqueda.

\subsection{Análisis de la información}

Se depura la información relevante para obtener procesos de transformación de material aglomerado, donde se extraen 11 procesos mostrados en la tabla 1 .

Tabla 1 Resumen de procesos de transformación.

\begin{tabular}{|c|c|}
\hline Proceso & Descripción \\
\hline $\begin{array}{l}\text { Hidrolizar el } \\
\text { aglutinante }\end{array}$ & $\begin{array}{l}\text { Mediante tratamiento con una } \\
\text { solución ácida para la placa de fibra } \\
\text { de pulverización, (Park Byeong-Dae, } \\
\text { 2016) }\end{array}$ \\
\hline $\begin{array}{l}\text { Método de } \\
\text { fusión }\end{array}$ & $\begin{array}{l}\text { Se realiza una mezcla en caliente, una } \\
\text { molienda de material para realizar } \\
\text { paneles de madera, (Barkoula, } \\
\text { Alcock, Cabrera, \& Peijs, 2008) }\end{array}$ \\
\hline $\begin{array}{l}\text { Fraccionamie } \\
\text { nto de fibra. }\end{array}$ & $\begin{array}{l}\text { Se realiza un proceso fibra seca, con } \\
\text { procesos de tamices (Benthien, } \\
\text { Heldner, \& Ohlmeyer, 2017). }\end{array}$ \\
\hline $\begin{array}{l}\text { Biodegradaci } \\
\text { ón de las } \\
\text { virutas }\end{array}$ & $\begin{array}{l}\text { El MDF se utiliza como mantillo para } \\
\text { el soporte estructural de suelos } \\
\text { agrícolas, (Cartmell, 2017) }\end{array}$ \\
\hline $\begin{array}{l}\text { Etiqueta } \\
\text { radiactiva de } \\
\text { carbono-14 }\end{array}$ & $\begin{array}{l}\text { La urea-formaldehido se puede } \\
\text { descomponer por biodegradación del } \\
\text { carbono orgánico, (Leungprasert \& } \\
\text { Otten, n.d.) }\end{array}$ \\
\hline Hidrólisis & $\begin{array}{l}\text { El colapso asociado de las partículas } \\
\text { de madera son causados por el vapor } \\
\text { de agua. (Ihnát, Lübke, Russ, \& } \\
\text { Borůvka, 2017) }\end{array}$ \\
\hline $\begin{array}{l}\text { Carbonizació } \\
\text { n }\end{array}$ & $\begin{array}{l}\text { convertir los materiales de madera en } \\
\text { carboncillos monolíticos. (Kercher \& } \\
\text { Nagle, 2002) }\end{array}$ \\
\hline Pirolisis & $\begin{array}{l}\text { Tres tipos de producto: carbón } \\
\text { activo, compuestos plásticos de } \\
\text { madera, nanocristales de celulosa, } \\
\text { (Zhong, Gu, Gao, Tu, \& Hu, 2017) }\end{array}$ \\
\hline Hidrotérmico & $\begin{array}{l}\text { Reconstituir tableros en paneles } \\
\text { nuevos por medio de condensación } \\
\text { de gases. }\end{array}$ \\
\hline $\begin{array}{l}\text { Bio- } \\
\text { rremediación }\end{array}$ & $\begin{array}{l}\text { Se tritura el material y se mezcla con } \\
\text { estiércol de aves de corral y residuos } \\
\text { verdes, (McMahon et al., 2008). }\end{array}$ \\
\hline $\begin{array}{l}\text { Calentamient } \\
\text { o óhmico }\end{array}$ & $\begin{array}{l}\text { Uso de corriente eléctrica, para } \\
\text { provocar un calentamiento rápido, } \\
\text { (Panel, 2012) }\end{array}$ \\
\hline
\end{tabular}




\section{Referencias Bibliografícas}

\section{Resultados}

En resultado, se obtienen 7 procesos químicos y 4 procesos físicos que están siendo utilizados en el mundo como recurso de reciclaje de los tableros aglomerado de madera, Figura 2.

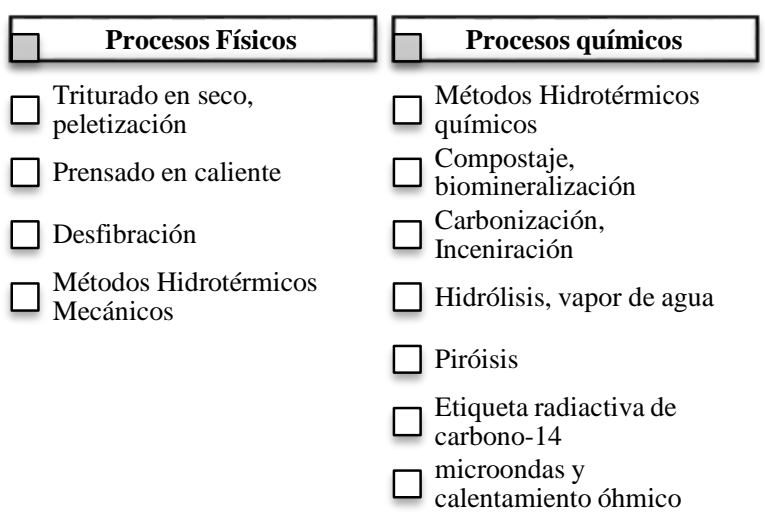

Fig. 2 Procesos para la transformación de tablero.

\section{Conclusiones}

Los resultados de la búsqueda han mostrado que existen investigaciones previas sobre procesos de transformación con fines de reciclaje de tableros de madera, con el fin de mitigar el impacto medio ambiental de estos residuos en vertederos o siendo utilizados como combustible.

La investigación generó como conclusión el análisis de 7 procesos químicos y 4 procesos físicos, los cuales serán estudiados en un trabajo futuro para el diseño de una adaptación tecnológica que supla las necesidades del sector del mueble y la madera en Colombianas.

\section{Agradecimientos}

A la Empresa Madecentro Colombia S.A,S, y a la Universidad Santo Tomás Bucaramanga por la contribución y apoyo en la investigación.
Barkoula, N. M., Alcock, B., Cabrera, N. O., \& Peijs, T. (2008). Fatigue properties of highly oriented polypropylene tapes and all-polypropylene composites. Polymers and Polymer Composites, 16(2), 101-113. https://doi.org/10.1002/pc

Benthien, J. T., Heldner, S., \& Ohlmeyer, M. (2017). Investigation of the interrelations between defibration conditions, fiber size and mediumdensity fiberboard (MDF) properties. European Journal of Wood and Wood Products, 75(2), 215-232. https://doi.org/10.1007/s00107-016$1094-2$

Cartmell, P. (2017). The Recycling of Waste MediumDensity Fibreboard Materials. The Recycling of Waste Medium-Density Fibreboard Materials, 1. Retrieved from https://homesteady.com/info8755091-recycling-waste-mediumdensityfibreboard-materials.html

Center, B. (2018). BioComposites Center. Retrieved from http://www.bc.bangor.ac.uk/

Costa, S., \& Teixeira, J. P. (2015). Formaldehyde: Human exposure, metabolism and potential health effects. In Formaldehyde: Synthesis, Applications and Potential Health Effects (pp. 1-29). Retrieved from https://www.scopus.com/inward/record.uri?eid $=2-\mathrm{s} 2.0-$

84956800177\&partnerID $=40 \& m d 5=$ f0b2c 9262 2bd3d9b691b5ab733469da2

Declaration, E. P. (2017). Environmental product declaration for medium density fibreboards (MDF) and for melamine faced medium density fibreboards ( MDF ).

Fronti de García, L., \& Cuesta Fernández, C. (2007). El protocolo de kioto y los costos ambientales. Revista Del Instituto Internacional de Costos, 1 , 9-31. Retrieved from http://dialnet.unirioja.es/descarga/articulo/3362 188.pdf

Ihnát, V., Lübke, H., Russ, A., \& Borůvka, V. (2017). Waste agglomerated wood materials as a secondary raw material for chipboards and fibreboards Part I. Preparation and characterization of wood chips in terms of their reuse. Wood Research, 62(1), 45-56. Retrieved from 
https://www.scopus.com/inward/record.uri?eid $=2-\mathrm{s} 2.0$ -

$85016978229 \&$ partnerID $=40 \& m d 5=61094465$ 2d3782653f2a75faadf10bc3

Kercher, A. K., \& Nagle, D. C. (2002). Evaluation of carbonized medium-density fiberboard for electrical applications. Carbon, 40(8), 13211330 . https://doi.org/10.1016/S00086223(01)00299-8

Leungprasert, S., \& Otten, L. (n.d.). Fate of Formaldehyde in MDF Sawdust during MSW Composting. Water.

McMahon, V., Garg, A., Aldred, D., Hobbs, G., Smith, R., \& Tothill, I. E. (2008). Composting and bioremediation process evaluation of wood waste materials generated from the construction and demolition industry. Chemosphere, 71(9), 1617-1628.

https://doi.org/10.1016/j.chemosphere.2008.01. 031

Panel, W. B. I. (2012). Recycling MDF: are we there yet? Recycling MDF: Are We There Yet?, 1. Retrieved from http://www.wbpionline.com/features/recyclingmdf-are-we-there-yet/

Park Byeong-Dae, R. R. M. (2016). Patent. Korean. Retrieved from https://patents.google.com/patent/KR10175785

8B 1/en?q $=($ mdf $) \& q=($ recycle $) \&$ before $=$ priority $: 20180$ $101 \&$ after $=$ priority: $20100101 \&$ oq $=(\mathrm{mdf})+($ recy cle)+before:priority:20180101+after:priority:20 100101

Primadera. (2016). Inauguración de Primadera, el nuevo fabricante de tableros MDP colombiano. Retrieved from http://www.notifix.info/es/noticiases/fabricantes-tableros/36024-inauguracion-deprimadera-el-nuevo-fabricante-de-tablerosmdp-colombiano

Zhong, R., Gu, J., Gao, Z., Tu, D., \& Hu, C. (2017). Impacts of urea-formaldehyde resin residue on recycling and reconstitution of wood-based panels. International Journal of Adhesion and Adhesives, 78, 60-66. https://doi.org/10.1016/j.ijadhadh.2017.06.019

A.K. Reyes Echeverría Diseñador Industrial de la Universidad Industrial de Santander - UIS; Maestría en iencias y Tecnologías Ambientales de la Universidad Santo Tomas, Bucaramanga. Profesor Universidad Manuela Beltrán.

L.E. Bautista Rojas Diseñador Industrial de la Universidad Industrial de Santander - UIS; Maestría en Ingeniería de Sistemas e Informática de la Universidad Industrial de Santander, (Actual) Doctorado en Ciencias de la Computación, Bucaramanga. Profesor Universidad Industrial de Santander.

L.C. Quintero Cárdenas.Diseñador Industrial de la Universidad Industrial de Santander - UIS.

* Para citar este artículo: Reyes Echeverría AK.,; Bautista Rojas LE.; Quintero Cárdenas LC. Analysis of technologies for the transformation of agglomerated wood material for recycling purposes.revista Bistua.2017.15(2):54-58.

+ Autor para el envió de correspondencia y la solicitud de las separatas: Reyes Echeverría AK. Universidad Santo Tomás, seccional Bucaramanga. akreyes42@gmail.com

Recibido: Octubre 29 de 2016

Aceptado: Febrero 02 de 2017 